\title{
Clasificación de frutos de tomates Cereza y Grape bajo diferentes sistemas de cultivo e intensidades de deshojado
}

\author{
Cherry and Grape tomato fruits classification according \\ different cropsystems and defoliate levels
}

\author{
Lais Perin ${ }^{*}$, Roberta Marins Nogueira Peil'1, Daniela Höhn ${ }^{1}$, Fabiane Kletke De Oliveira ${ }^{1}$, \\ Eduardo Anibele Streck ${ }^{1}$, Albertina Radtke Wieth ${ }^{2}$, Thiago Freitas Da Luz y yaulo Roberto Grolli ${ }^{1}$
}

\begin{abstract}
RESUMEN
Con el aumento de la superficie cultivada de tomates de pequeño diámetro, es necesario establecer una normativa de clasificación para su comercialización. El objetivo del estudio es proponer una clasificación para tomates tipos Cereza y Grape en función al tamaño de los frutos, el efecto del sistema de cultivo y la intensidad del deshojado. Se cultivaron tomates tipo cereza Wanda y tipo Grape Dolcetto, bajo dos sistemas cerrados de cultivo sin suelo (canales y macetas). Se utilizó cáscara de arroz in natura como sustrato, con tres intensidades de deshojado (plantas con el simpodio completo; una y dos hojas eliminadas por simpodio). Para la clasificación de los frutos, se utilizó dos series de cinco tamices con mallas de perforaciones adaptadas a cada variedad. Se seleccionaron las siguientes mallas: para Cereza 35, 30, 25, $20 \mathrm{~mm}$ y recolector, y para Grape 25, 20, 15, $10 \mathrm{~mm}$ y recolector. A partir del inicio de la recolección de los frutos se han evaluado las variables número y peso medio de los frutos retenidos en cada uno de los tamices para cada una de las variedades. Se evaluaron 8.284 frutos del tipo Cereza y 8.033 del tipo Grape durante siete meses de cosecha. Los datos sometidos a un análisis de varianza, regresión lineal; las medias fueron comparadas con la prueba de Tukey. Los resultados indicaron que el deshojado disminuyó la cantidad de frutos clasificados como grandes para ambas variedades. El sistema de canales favoreció la producción de frutos clasificados como grandes para la variedad Grape. Los tamices 1, 2 y 3 fueron adecuados para la clasificación de los frutos de ambas variedades. Es posible ordenar el tamaño de frutos de tomates con el uso de tamices específicos para cada tipo de variedad.
\end{abstract}

Palabras clave: canales, macetas, peso medio, Solanum lycopersicum, tamices.

\begin{abstract}
With the advancement of small size tomato cultivation in recent years, studies on fruit grading are necessary to obtain a commercialization standard. Therefore, the objective of the work was to perform the grading, based on the fruit size of Grape and Cherry mini tomatoes, considering the effects of different cropping systems and defoliation levels. Tomato plants of Wanda Cherry Hybrid ${ }^{\circledR}$ and Dolcetto Grape Hybrid ${ }^{\circledR}$ varieties were grown in two closed soilless systems (troughs and pots), using substrate of raw rice husk, under three different defoliation intensities (without defoliation, removal of one and two leaves of the sympodium). For fruits grading, two sets of five sieves were used, with mesh drilling adapted for each variety. The chosen meshes were: 35, 30, 25, 20 and $0 \mathrm{~mm}$ for Cherry, and 25,20,15, 10 and $0 \mathrm{~mm}$ for Grape. From the beginning of the fruit harvest the variables of number and mean weight of fruits retained in each of the sieves were evaluated, for each variety. A total of 8284 fruits of the Cherry type and 8033 fruits of the Grape type were evaluated during seven months of harvest. Data were submitted to analysis of variance, linear regression, and comparison of means by the Tukey test. The obtained results indicated that defoliation reduced the amount of fruits grading as large for both Cherry and Grape varieties. The troughs system benefited the production of Grape variety fruits grading as large. The sieves 1, 2 and 3 are suitable for the fruits grading of both varieties. The grading of mini tomato fruits with the use of specific sieves for each type variety is feasible.
\end{abstract}

Key words: troughs, pot, mean weight, Solanum lycopersicum, sieves.

\footnotetext{
$1 \quad$ Universidade Federal de Pelotas, Pelotas/RS, Brazil.

2 Universidade Federal do Rio Grande do Sul, Porto Alegre/RS, Brazil.

* Autor por correspondencia: laisp.agro@gmail.com
}

Fecha de Recepción: 11 julio, 2017.

Fecha de Aceptación: 10 enero, 2018.

DOI: http://dx.doi.org/10.4067/S0718-34292018005000603. Publicado en línea: 24-agosto-2018. 


\section{Introducción}

Entre los grupos de tomate (Solanum lycopersicum L.), los tomates de pequeño diámetro han conquistado un espacio en el mercado de consumo debido a su tamaño y agradable sabor (Beckles, 2012). Las plantas de estos tomate son de alta productividad, alcanzando cerca de 22 kg planta $^{-1}$ año $^{-1}$ (Mello, 2016). Además de su productividad, los frutos alcanzan precios mayores al $20 \%$, respecto a los tomates convencionales (Caron et al., 2013).

Con la finalidad de garantizar una mayor calidad de los frutos, se han buscado alternativas de manejo del cultivo que favorezcan el desarrollo de la planta y de los frutos, siendo el deshojado una práctica recomendada. El deshojado reduce la superficie foliar y, por ende, busca proporcionar un equilibrio entre el crecimiento vegetativo y reproductivo de la planta, a través de una adecuada relación fuente/sumidero (Radin et al., 2008). Además de la mejoría en la producción y calidad, el deshojado mejora la ventilación del dosel del cultivo.

Normalmente, la producción de minitomates se hace bajo invernadero, empleando macetas con sustrato como sistema de cultivo. Sin embargo, en los últimos años, los productores están cambiando las macetas por canales de cultivo, dentro de los cuales se dispone directamente el sustrato. Ambos sistemas son abiertos o a disolución perdida, o sea, la solución nutritiva drenada es lixiviada al medio ambiente. La producción de tomate en sistemas "cerrados" de cultivo es una alternativa siempre y cuando se cuente con agua de buena calidad. La disolución nutritiva drenada es colectada y reutilizada, lo que disminuye la contaminación ambiental, además de mejorar la eficiencia en el uso del agua y los fertilizantes, bajando los costos del sistema. En este caso, el sustrato debe ser inerte o con baja capacidad de intercambio catiónico (CIC). La cáscara de arroz in natura es un sustrato con alto potencial que se puede emplear con éxito en este cultivo, como fue observado con anterioridad por Peil et al. (2014).

Respecto a la clasificación de los frutos, los grupos de tomate de mesa tienen normas de clasificación establecidas por la Companhia de Entrepostos e Armazéns Gerais de São Paulo CEAGESP (2003). Sin embargo, para tomates de pequeño calibre, no se han definido las normas de clasificación en cuanto al tamaño y peso medio para fines de comercialización. Sin embargo, existen dos normativas internacionales que clasifican este tipo de tomate, CODEX (2006) y UNECE (2012). El Codex define como un requisito el tamaño mínimo de $15 \mathrm{~mm}$ de diámetro para los tomates Cereza y Cóctel, y no se puede encontrar más de $10 \%$ de los frutos con tamaño $5 \mathrm{~mm}$ mayor o menor que la clase definida. La Unece determina el tamaño del fruto por el diámetro máximo de la sección transversal, siendo la tabla de clasificación opcional para tomates cereza y cóctel por debajo de $40 \mathrm{~mm}$ de diámetro.

La clasificación es importante para los agricultores porque permite la estandarización del producto y un mayor valor añadido. En el ámbito académico, las normas de clasificación se utilizan para evaluar los efectos del manejo de un cultivo sobre las variables de estudio. El objetivo del estudio es proponer una clasificación para tomates tipos Cereza y Grape en función al tamaño de los frutos, el efecto del sistema de cultivo y la intensidad del deshojado.

\section{Materiales y Métodos}

El trabajo se llevó a cabo entre el 31 de agosto del 2015 al 31 de mayo del 2016, en el Campo Didático e Experimental del Departamento de Fitotecnia, de la Faculdad de de Agronomía Eliseu Maciel, en el Campus de la Universidade Federal de Pelotas (31 $\left.52^{\circ} \mathrm{S}-52^{\circ} 21^{\prime} \mathrm{W}\right)$, ubicado en la ciudad de Capão do Leão, estado de Rio Grande do Sul, Brasil.

El ensayo se realizó en un invernadero modelo "Techo en arco" de estructura metálica con una superficie de $210 \mathrm{~m}^{2}$ (10 x $\left.21 \mathrm{~m}\right)$ y 4,5 m de altura máxima, dispuesto en la dirección Norte-Sur y cubierta de polietileno de baja densidad $(150 \mu \mathrm{m}$ de espesor). El control ambiental del invernadero se efectuó únicamente por ventilación natural abriendo y cerrando diariamente las cortinas laterales y las puertas según las condiciones atmosféricas.

La disolución nutritiva utilizada fue adaptada de Rocha, Peil y Cogo (2010), cuya composición de macronutrientes (mmol L-1), fue: 12,2 de $\mathrm{NO}_{3}{ }^{-}$; 1,5 de $\mathrm{H}_{2} \mathrm{PO}_{4}^{-} ; 2,25$ de $\mathrm{SO}_{4}^{-2} ; 1,2 \mathrm{de} \mathrm{NH}_{4}^{+} ; 6,0 \mathrm{de}$ $\mathrm{K}^{+} ; 3,5 \mathrm{de} \mathrm{Ca}^{+2} ; 2,0$ de $\mathrm{Mg}^{+2} ; \mathrm{y}$ micronutrientes (mg $\mathrm{L}^{-1}$ ): 3,0 de Fe; 0,5 de Mn; 0,05 de Zn; 0,15 de B; 0,02 de $\mathrm{Cu}$ y 0,01 de Mo. Para la preparación de la disolución nutritiva, se utilizó agua de lluvia con 
conductividad eléctrica (CE) de $0,0 \mathrm{dS} \mathrm{m}^{-1}$. La CE de la disolución se midió con eletrocondutivímetro digital y se obtuvo el valor de $1,82 \mathrm{dSm}^{-1}$.

Se utilizaron semillas de tomate de las variedades Cereza Híbrido Wanda ${ }^{\circledR}$ y Grape Híbrido Dolcetto ${ }^{\circledR}$ (Isla Sementes). La siembra se realizó el 20 de julio del 2015, en bandejas multicelulares de 128 células conteniendo sustrato comercial Carolina Soil, las cuales se pusieron en un sistema flotante para fertirrigación. Cuando las plantas presentaban cuatro hojas verdaderas, se despuntó la yema apical con el fin de estimular el crecimiento de los tallos laterales, seleccionándose dos tallos laterales por planta para la conducción del cultivo.

El trasplante se realizó siete días después del despunte del tallo principal. La base para la instalación de los dos sistemas de cultivo en sustrato consistió en 12 canales de madera (7,5 m de longitud y 0,30 $\mathrm{m}$ de ancho), dispuestos en 6 líneas dobles, con distancia de $0,50 \mathrm{~m}$ y un pasillo de 1,2 $\mathrm{m}$ de ancho. Los canales fueron recubiertos internamente con polietileno flexible, blanco-negro, de $200 \mu \mathrm{m}$ de espesor y sujetados por apoyos de madera. La pendiente fue del $3 \%$ para favorecer la circulación de la disolución nutritiva hacia el estanque colector de la solución. De este modo, se estableció un sistema de cultivo cerrado con reutilización de la solución nutritiva lixiviada.

El primer sistema de cultivo se hizo en macetas de plástico, donde se colocaron 7 litros de sustrato hasta una altura de 0,22 m. En cada maceta, se trasplantó una planta de tomate. Se colocaron 19 macetas por canal. El segundo sistema de cultivo fue nombrado de "sistema de canales", en el cual se depositó el sustrato directamente sobre el canal de plástico, en una capa de 7,5 $\mathrm{m}$ de largo, 0,27 $\mathrm{m}$ de ancho y 0,08 $\mathrm{m}$ de altura, lo que resultó en 8,5 litros de sustrato para cada planta. El sustrato utilizado en ambos sistemas fue cáscara de arroz in natura que surgió del proceso de separación del grano de arroz en la industria de procesamiento.

Antes del trasplante, el sustrato fue saturado con agua para el lavado. Tras el trasplante, se regó con disolución nutritiva. El aporte del fertirriego se hizo con cintas de goteo, con caudal de 1,6 $\mathrm{L} \mathrm{hora}^{-1}$ y distancia entre goteros de $0,40 \mathrm{~m}$. El aporte de la disolución nutritiva se hizo según la demanda de las plantas y las condiciones atmosféricas, alcanzando riegos de $30 \mathrm{~min}$ (en intervalos de una hora) de 7:00 am a 7:00 pm. Durante la noche, se realizó un riego de 15 minutos a la 1:00 am.
La disolución nutritiva se controló diariamente por medio de mediciones de conductividad eléctrica (mediante un eletrocondutivímetro digital) y el $\mathrm{pH}$ (empleando un medidor de $\mathrm{pH}$ digital) de la solución drenada. El pH se mantuvo entre 5,5 y 6,5 a través de la adición de solución de ácido sulfúrico $\left(\mathrm{H}_{2} \mathrm{SO}_{4} 1 \mathrm{~N}\right)$ o hidróxido de sodio $(\mathrm{NaOH} 1 \mathrm{~N})$. $\mathrm{La}$ reposición de nutrientes o de agua se llevó a cabo por medio de la adición de soluciones concentradas o agua de lluvia almacenada, manteniéndose el valor de la conductividad eléctrica de $1,8 \mathrm{dSm}^{-1}$.

Las plantas se dispusieron de modo que la distancia entre ellas en la línea de cultivo fue de $0,40 \mathrm{~m}$, lo que corresponde a una densidad de 2,9 plantas $\mathrm{m}^{-2}$, en un total de 19 plantas por canal, independientemente del sistema empleado. El entutorado se realizó a través de un hilo de plástico de $8 \mathrm{~m}$ de longitud enrollado alrededor de ganchos suspendidos a 3,0 m por encima de la línea de cultivo y sujetado por la estructura del invernadero.

Tras la aparición del primer racimo floral, se inició la práctica del deshojado, y se continuó a medida que salían nuevos racimos florales durante todo el ciclo de cultivo (nueve meses). El deshojado se hizo en tres niveles distintos: nivel 0 - ninguna hoja removida, manteniendo el simpodio completo (dos hojas por debajo y una hoja por encima de la inflorescencia); nivel 1 - eliminación de una hoja por simpodio, mediante la retirada de la hoja "a" debajo de la inflorescencia; y nivel 2- eliminación de dos hojas por simpodio, mediante la retirada de las hojas "a" y "c", quedando únicamente la hoja "b" (Figura 1). Al final de la recolección de los frutos de cada racimo, se ha practicado el deshojado de las hojas situadas debajo del racimo cosechado. Las demás prácticas culturales y fitosanitarias se han llevado a cabo en la medida en que se necesitaban.

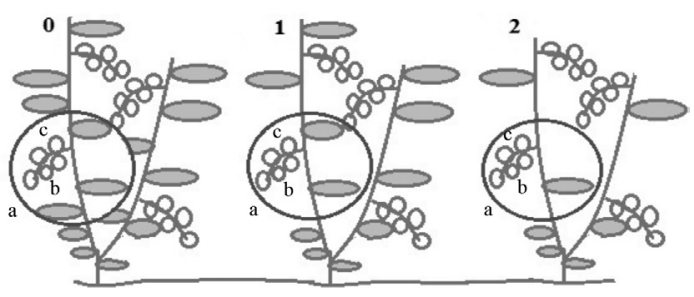

Figura 1. Niveles de deshojado en el cultivo de tomate. 0) Simpodio completo, sin deshojado. 1) Una hoja eliminada por simpodio. 2) Dos hojas eliminadas por simpodio. 
Para la clasificación de los frutos, se prepararon cinco tamices, empleándose para ello cajas de cartón con dimensiones de 36 × 26 × $5 \mathrm{~cm}$. Las perforaciones se hicieron de forma manual utilizando un cuchillo afilado y de acuerdo con el formato de cada tipo de tomate. Para los tomates Cereza se utilizaron las cajas recomendadas por Fernandes et al. (2007), considerando la forma redonda del fruto para la fabricación de las mallas de los tamices. En cuanto a los tomates Grape, no se encontraron referencias respecto al tipo de cajas, por lo cual se ha desarrollado una serie de cinco tamices, pero con diferentes perforaciones, con forma rectangular debido a la forma oblonga de los frutos. Las mallas elegidas para la fabricación de los tamices fueron: para Cereza 35, 30, 25, $20 \mathrm{~mm}$ y el colector, y para Grape 25, 20, 15, $10 \mathrm{~mm}$ y colector, que se refieren respectivamente a los tamices 1, 2, 3, 4 y 5 (Figura 2). Se consideraron como frutos comerciales, de buena calidad, aquellos retenidos en los tamices 1,2 y 3 . Los frutos de los tamices 4 y 5 (colectora) fueron considerados descarte.

La recolección empezó a los 81 días tras el trasplante, el 20 de noviembre del 2015, y se extendió hasta el 31 de mayo del 2016. La clasificación de los frutos se realizó durante los siete meses que duró la cosecha. Todos los frutos recolectados de las plantas fueron pasado por los tamices, contados y pesados para obtener el número y el peso medio de frutos retenido en cada tamiz.

El diseño experimental fue de bloques al azar con tres repeticiones en parcelas sub-sub divididas. El factor sistema de cultivo (canales y macetas) se dispuso en tres parcelas resultando un total de 144 plantas evaluadas (24 plantas sistema $^{-1}$ en cada parcela). El factor tipo (Cereza y Grape) fue asignado como sub-parcela (12 plantas variedad $^{-1}$ ) y el factor deshojado (niveles 0,1 y 2 ) se asignó como la sub-sub parcela (4 plantas nivel ${ }^{-1}$ variedad $\left.^{-1}\right)$. Los datos se sometieron a análisis de varianza, regresión lineal, y la comparación

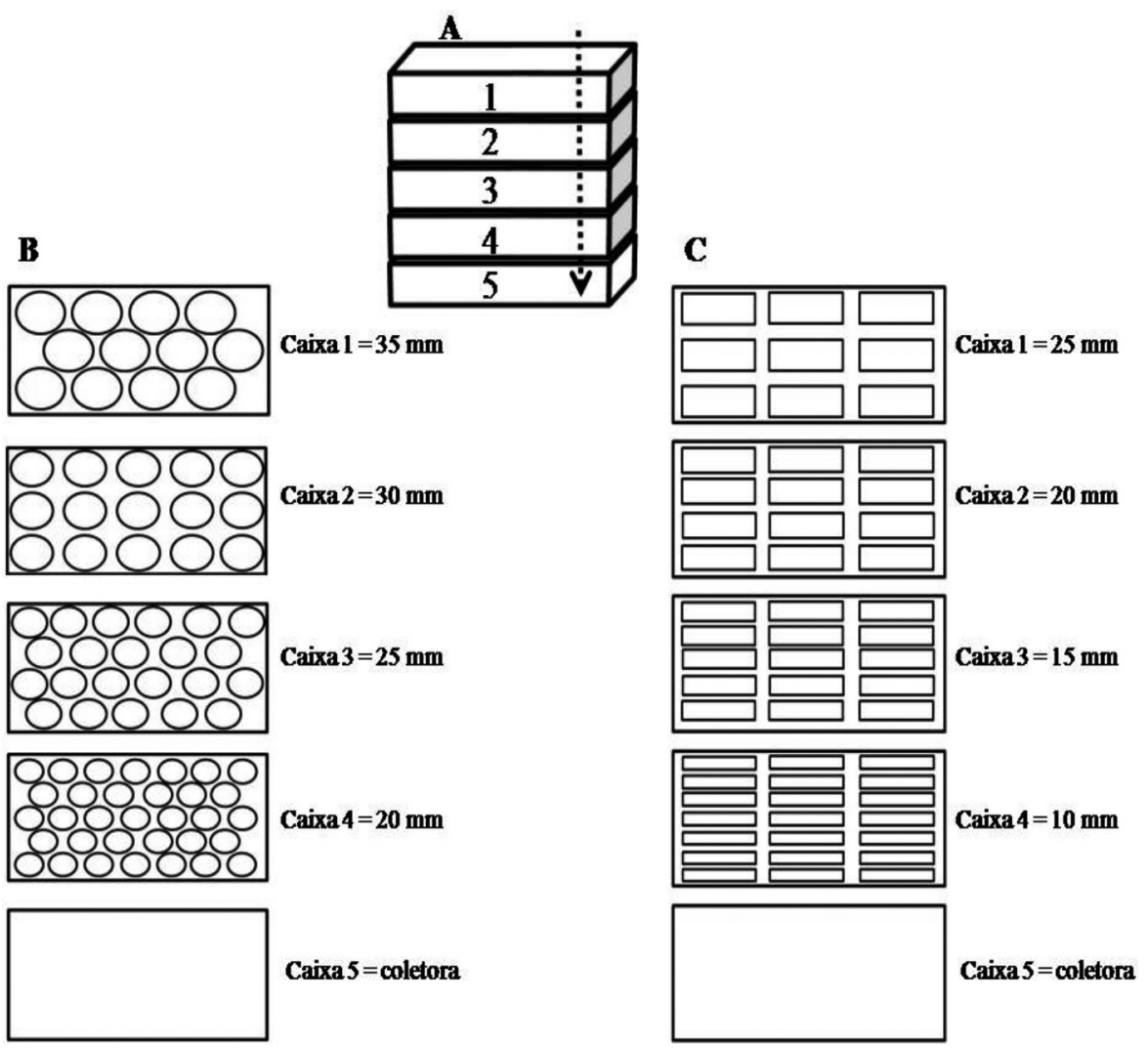

Figura 2. Tamices utilizados para clasificación de tomates en orden del mayor al menor diámetro o tamaño de las perforaciones. A: Tamices superpuestos; B: Cajas para clasificación de tomate tipo Cereza y C: Cajas de clasificación de tomate tipo Grape. 
de medias mediante la prueba de Tukey al 5\% de probabilidad utilizando el software GENES (Cruz, 2013).

\section{Resultados y Discusión}

Se observó que el sistema afectó significativamente el número de frutos en la variedad Grape. En cambio, el deshojado influyó en el número de frutos de la variedad Cereza únicamente. El factor tamiz fue altamente significativo para todas las variables analizadas, en ambas variedades (Tabla 1).

No hubo interacción significativa entre sistema $\mathrm{x}$ deshojado $\mathrm{y}$ entre sistema $\mathrm{x}$ deshojado $\mathrm{x}$ tamiz para cualquiera de las variables analizadas en ambas variedades. Para la variedad Cereza hubo una interacción significativa para la variable número de frutos entre los factores deshojado $\mathrm{x}$ tamiz. Mientras que para la variedad Grape hubo una interacción significativa para número de frutos entre los factores sistema $\mathrm{x}$ tamiz y entre deshojado $\mathrm{x}$ tamiz. El coeficiente de variación (CV) fluctuó del 12,67 al 38,19\% (Tabla 1). Todas las variables presentaron una distribución normal de los errores por la prueba de Shapiro-Wilk (w $>$ p).

El sistema de cultivo en canales no mostró diferencias significativas con relación al cultivo en macetas para ninguna de las variables evaluadas en la variedad Cereza. Sin embargo, para la variedad Grape, este sistema ha permitido el desarrollo de un mayor número de frutos (Tabla2). Esta variación puede ser debido a las características genotípicas de cada cultivar, que resultan en formas y tamaños distintos de los frutos y diferencias en el crecimiento de la raíz, lo que implica diferencias en la absorción de agua y nutrientes $\mathrm{y}$, consecuentemente, en distintas respuestas de las dos variedades a los sistemas de cultivo.

La práctica del deshojado puede llevarse a cabo sin perjuicio al peso medio y al número de frutos solamente en las plantas de la variedad Grape (Tabla 2). El deshojado ofrece algunas ventajas, tales como el incremento de la ventilación del dosel y la disminución de la ocurrencia de plagas y enfermedades (Kanyomeka y Shivute, 2005). Sin embargo, esta práctica requiere más mano de obra. En cambio, para la variedad Cereza, el deshojado afectó el número de frutos producidos. Presumiblemente, este efecto puede ser atribuido al mayor tamaño de los frutos de esta variedad, lo que indica una más elevada demanda de foto asimilados y una mayor superficie foliar para garantizar un mayor cuajado de los frutos en comparación con la de la variedad Grape que produjo frutos de menor peso medio.

El peso medio de los frutos disminuyó progresivamente en la medida en que la malla del tamiz disminuyó (Tabla2). La mayoría de los frutos se ha clasificado en los tamices 2 y 3 . Los frutos más grandes, retenidos en el primer tamiz, se han obtenido, especialmente, en la primera recolección,

Tabla 1. Cuadrados medios del análisis de varianza (ANOVA) para las variables número de frutos clasificados por tamiz y peso medio de frutos en cada tamiz con respecto a los factores sistema

(Sist), deshojado (Desh) y tamiz y sus interacciones para dos variedades de tomates.

\begin{tabular}{lccccc}
\hline \multirow{2}{*}{ Factores } & \multicolumn{2}{c}{ Cereza Wanda } & & \multicolumn{2}{c}{ Grape Dolcetto } \\
\cline { 2 - 3 } \cline { 6 - 7 } & $N^{\mathrm{o}}$ Frutos & Peso Medio & & $N^{\mathrm{o}}$ Frutos & Peso Medio \\
\hline Sist & $35^{\mathrm{ns}}$ & $0,113^{\mathrm{ns}}$ & & $7200^{* *}$ & $0,032^{\mathrm{ns}}$ \\
Desh & $5059^{* * *}$ & $1,908^{\mathrm{ns}}$ & & $1150^{\mathrm{ns}}$ & $1,041^{\mathrm{ns}}$ \\
Tamices & $170079^{* * *}$ & $973,311^{* * *}$ & & $203295^{* * *}$ & $429,445^{* * *}$ \\
Sist*Desf & $105^{\mathrm{ns}}$ & $1,806^{\mathrm{ns}}$ & & $298^{\mathrm{ns}}$ & $0,523^{\mathrm{ns}}$ \\
Sist*Tamiz & $903^{\mathrm{ns}}$ & $2,421^{\mathrm{ns}}$ & & $3094^{*}$ & $0,013^{\mathrm{ns}}$ \\
Desh*Tamiz & $5890^{* * *}$ & $1,187^{\mathrm{ns}}$ & & $2429^{*}$ & $1,547^{\mathrm{ns}}$ \\
Sist*Desh*Tamiz & $211^{\mathrm{ns}}$ & $2,232^{\mathrm{ns}}$ & & $414^{\mathrm{ns}}$ & $1,757^{\mathrm{ns}}$ \\
\hline Error & 616 & 1,833 & & 1127 & 0,975 \\
\hline Media & 92,04 & 10,69 & & 87,92 & 6,32 \\
\hline CV\% & 26,96 & 12,67 & 38,19 & 15,62 \\
\hline
\end{tabular}

* significativo $(\mathrm{p}<0,05)$; $*$ significativo $(\mathrm{p}<0,01)$; *** significativo $(\mathrm{p}<0,001)$; ${ }^{\text {ns }}$ no significativo $(\mathrm{p}>0,05)$. 
Tabla 2. Efecto de los factores sistema de cultivo, nivel de deshojado y tamiz de clasificación sobre el número de frutos y el peso medio de frutos de dos variedades de tomates.

\begin{tabular}{|c|c|c|c|c|}
\hline \multirow{2}{*}{ Factores } & \multicolumn{2}{|c|}{ Cereza } & \multicolumn{2}{|c|}{ Grape } \\
\hline & $\mathrm{N}^{\circ}$ frutos tamiz ${ }^{-1}$ & Peso Medio ( $\mathrm{g}_{\text {fruto }}{ }^{-1}$ ) & $\mathrm{N}^{\mathrm{o}}$ frutos tamiz ${ }^{-1}$ & Peso Medio ( $\mathrm{g}$ fruto ${ }^{-1}$ ) \\
\hline \multicolumn{5}{|l|}{ Sistema } \\
\hline Canaletas & $92,68^{\mathrm{ns}}$ & $10,72^{\text {ns }}$ & $96,87 \mathrm{~A}$ & $6,34^{\mathrm{ns}}$ \\
\hline Macetas & 91,42 & 10,65 & $78,98 \mathrm{~B}$ & 6,30 \\
\hline \multicolumn{5}{|l|}{ Deshojado } \\
\hline 0 & $104,87 \mathrm{~A}$ & $10,90^{\text {ns }}$ & $92,03^{\mathrm{ns}}$ & $6,30^{\mathrm{ns}}$ \\
\hline 1 & $92,37 \mathrm{AB}$ & 10,76 & 90,93 & 6,52 \\
\hline 2 & $78,90 \mathrm{~B}$ & 10,41 & 80,8 & 6,15 \\
\hline \multicolumn{5}{|l|}{ Tamiz } \\
\hline 1 & $10,28 \mathrm{D}$ & $21,38 \mathrm{~A}$ & $6,28 \mathrm{C}$ & $13,28 \mathrm{~A}$ \\
\hline 2 & $158,28 \mathrm{~B}$ & $14,12 \mathrm{~B}$ & $175,50 \mathrm{~B}$ & $8,72 \mathrm{~B}$ \\
\hline 3 & $230,44 \mathrm{~A}$ & $9,69 \mathrm{C}$ & $228,56 \mathrm{~A}$ & $5,71 \mathrm{C}$ \\
\hline 4 & $33,78 \mathrm{C}$ & $5,38 \mathrm{D}$ & $27,33 \mathrm{C}$ & $3,00 \mathrm{D}$ \\
\hline 5 & $27,44 \mathrm{CD}$ & $2,87 \mathrm{E}$ & $1,94 \mathrm{C}$ & $0,88 \mathrm{E}$ \\
\hline Media & 92,04 & 10,69 & 87,92 & 6,32 \\
\hline $\mathrm{CV} \%$ & 26,96 & 12,67 & 38,19 & 15,62 \\
\hline
\end{tabular}

Medias seguidas por la misma letra en la columna no difieren estadísticamente entre sí por la prueba de Tukey $(\mathrm{p}<0,05)$;

ns: no significativo al $5 \%$ de probabilidad;

Tamiz para Cereza $1=35 \mathrm{~mm}, 2=30 \mathrm{~mm}, 3=25 \mathrm{~mm}, 4=20 \mathrm{~mm}$ y $5=$ colectora;

Tamiz para Grape $1=25 \mathrm{~mm}, 2=20 \mathrm{~mm}, 3=15 \mathrm{~mm}, 4=10 \mathrm{~mm}$ y $5=$ colectora.

sobre todo para el tipo Grape. La mayor parte de los frutos no comerciales se obtuvieron en las últimas cosechas. La irregularidad del tamaño de los frutos puede ser atribuida, inicialmente, al efecto de las bajas temperaturas en las primeras cosechas a finales de la primavera y luego, desde la mitad hasta el final del ciclo, con la salida del verano y la entrada del otoño, como resultado de la menor radiación solar. Según Alvarenga (2013), temperaturas por debajo del rango adecuado (entre 14 y $25^{\circ} \mathrm{C}$ ) durante largos períodos de tiempo y poca intensidad lumínica pueden causar daños a la floración y polinización.

Los datos presentados en la Tabla 3 indican que el deshojado disminuyó el número de frutos en el tamiz 2 de ambas variedades. En la clasificación de los frutos pertenecientes a las plantas que permanecieron con simpodio completo, el número de frutos clasificados en los tamices 2 y 3 fue similar. Mientras que, en las plantas que han sufrido el deshojado (una o dos hojas), el número de frutos en el tamiz 2 fue significativamente menor en relación con el tamiz 3. No hubo diferencia significativa en el número de frutos clasificados en tamices 1, 4 y 5 de ambas variedades.
La Tabla 3 muestra que el deshojado disminuyó la cantidad de frutos grandes. Así, mantener el simpodio completo, el cual es compuesto por las dos hojas situadas debajo y la hoja ubicada arriba de la inflorescencia (Chamarro, 1995), es necesario para mejorar el tamaño de los frutos y, consecuentemente, para una buena aceptación en el mercado. Sin embargo, hay que poner de manifiesto el efecto beneficioso del deshojado solamente de la parte basal del tallo, por debajo de los racimos ya cosechados o en recolección, sin ocasionar daño a la producción y calidad de los frutos, mejorando el manejo y facilitando el control de plagas y enfermedades (Kim et al., 2014; Silva et al., 2011).

Para los frutos de las plantas de la variedad Cereza sin deshojado y con una hoja removida por simpodio, los datos de peso medio se han diferenciado de forma significativa entre los tamices 1,2 y 3 . Sin embargo, no mostraron diferencias significativas entre los tamices 4 y 5 (Tabla3). En las plantas con dos hojas retiradas por simpodio, el peso medio de los frutos se diferenció entre cada uno de los tamices.

Para los frutos de las plantas de la variedad Grape con una o dos hojas retiradas, los datos de 
Tabla 3. Interacción entre factores deshojado y tamiz (Desh*Tam) para las variables número de frutos clasificados en cada tamiz y peso medio de los frutos en cada tamiz para dos variedades de tomates.

\begin{tabular}{|c|c|c|c|c|c|}
\hline \multirow{2}{*}{\multicolumn{2}{|c|}{$\begin{array}{l}\text { Interacción } \\
\text { Desh*Tam }\end{array}$}} & \multicolumn{2}{|c|}{ Cereza Wanda } & \multicolumn{2}{|c|}{ Grape Dolcetto } \\
\hline & & $\mathrm{N}^{\circ}$ frutos tamiz ${ }^{-1}$ & Peso Medio ( $\mathrm{g}$ fruto $^{-1}$ ) & $\mathrm{N}^{\circ}$ frutos tamiz ${ }^{-1}$ & Peso Medio ( $\mathrm{g}_{\text {fruto }}-1$ ) \\
\hline \multirow{5}{*}{0} & 1 & $14,50 \mathrm{D}$ & $21,28 \mathrm{~A}$ & $6,17 \mathrm{C}$ & $13,28 \mathrm{~A}$ \\
\hline & 2 & $225,83 \mathrm{~A}$ & $14,67 \mathrm{~B}$ & $211,33 \mathrm{~A}$ & $9,20 \mathrm{~B}$ \\
\hline & 3 & $214,83 \mathrm{~A}$ & $10,09 \mathrm{C}$ & $205,50 \mathrm{AB}$ & $5,61 \mathrm{C}$ \\
\hline & 4 & $33,83 \mathrm{D}$ & $5,45 \mathrm{D}$ & $29,83 \mathrm{C}$ & $2,43 \mathrm{DE}$ \\
\hline & 5 & $35,33 \mathrm{D}$ & $3,00 \mathrm{DE}$ & $1,83 \mathrm{C}$ & $1,00 \mathrm{E}$ \\
\hline \multirow{5}{*}{1} & 1 & $9,17 \mathrm{D}$ & $21,21 \mathrm{~A}$ & $8,17 \mathrm{C}$ & $14,17 \mathrm{~A}$ \\
\hline & 2 & $157,50 \mathrm{~B}$ & $14,59 \mathrm{~B}$ & $178,17 \mathrm{AB}$ & $8,54 \mathrm{~B}$ \\
\hline & 3 & $235,33 \mathrm{~A}$ & $9,81 \mathrm{C}$ & $244,17 \mathrm{~A}$ & $5,77 \mathrm{C}$ \\
\hline & 4 & $36,17 \mathrm{D}$ & $5,22 \mathrm{DE}$ & $27,00 \mathrm{C}$ & $3,17 \mathrm{D}$ \\
\hline & 5 & $23,67 \mathrm{D}$ & $2,95 \mathrm{DE}$ & $2,67 \mathrm{C}$ & $0,93 \mathrm{E}$ \\
\hline \multirow{5}{*}{2} & 1 & $7,17 \mathrm{D}$ & $21,64 \mathrm{~A}$ & $4,50 \mathrm{C}$ & $12,44 \mathrm{~A}$ \\
\hline & 2 & $91,50 \mathrm{C}$ & $13,11 \mathrm{~B}$ & $137,00 \mathrm{~B}$ & $8,43 \mathrm{~B}$ \\
\hline & 3 & $241,17 \mathrm{~A}$ & $9,15 \mathrm{C}$ & $236,00 \mathrm{~A}$ & $5,74 \mathrm{C}$ \\
\hline & 4 & $31,33 \mathrm{D}$ & $5,47 \mathrm{D}$ & $25,17 \mathrm{C}$ & $3,39 \mathrm{D}$ \\
\hline & 5 & $23,33 \mathrm{D}$ & $2,66 \mathrm{E}$ & $1,33 \mathrm{C}$ & $0,72 \mathrm{E}$ \\
\hline \multicolumn{2}{|c|}{ Media } & 92,04 & 10,69 & 87,92 & 6,32 \\
\hline \multicolumn{2}{|c|}{$\mathrm{CV} \%$} & 26,96 & 12,67 & 38,19 & 15,62 \\
\hline
\end{tabular}

Medias seguidas de misma letra en la columna no difieren estadísticamente entre sí pela prueba de Tukey $(\mathrm{p}<0,05)$.

peso medio mostraron diferencias significativas entre todos los tamices. En cambio, para las plantas sin deshojado, los frutos clasificados en los tamices 4 y 5 presentaron pesos medios similares.

Para la variedad Grape, la interacción sistema $\mathrm{x}$ tamiz fue significativa con $\mathrm{p}<0,05$ (Tabla4). El sistema de canales ha incrementado el desarrollo de un mayor número de frutos clasificados en el tamiz 2, mientras que el sistema de macetas aumentó el número de frutos en el tamiz 3 . El peso medio de los frutos respondió de forma negativa a la disminución del tamaño de la malla de los tamices de clasificación para ambos sistemas de cultivo.

Teniendo en cuenta solamente la clasificación general (Tabla 5), se obtuvo como resultado una mayor concentración de frutos en la clase mediana, con 50,07 y $51,21 \%$, seguido de la clase grande, con 34,39 y $39,32 \%$ para Cereza y Grape, respectivamente. Las clases de frutos que presentaron los menores porcentajes, del $2,23 \%$, fueron los frutos considerados gigantes para la variedad Cereza y los frutos considerados desechos para Grape con 1,18\%. Menezes et al. (2012) obtuvieron resultados similares al evaluar la variedad Carolina del tipo Cereza en sistema de producción orgánico, logrando un mayor porcentaje de frutos medianos que de frutos gigantes.

La clasificación de los frutos de tomate mostró respuestas similares a las obtenidas por Fernandes et al. (2007), presentando una variación de menos de dos gramos en el peso medio correspondiente a cada clase. Para los frutos de clase gigante se obtuvieron pesos medios superiores a $18 \mathrm{~g}$; para los frutos grandes, entre 13 y $18 \mathrm{~g}$; la clase mediana entre 8 y $13 \mathrm{~g}$; y los frutos pequeños entre 5 y $8 \mathrm{~g}$ (Tabla 5). Mazuela et al. (2012) en su investigación observaron pesos medios de frutos que fueron desde el 9,8 hasta el 10,6 $\mathrm{g}$ fruto $^{-1}$ con diámetros de frutos entre 25 y $27 \mathrm{~cm}$. Estos frutos representan la clase referente al tamiz 3 y concuerdan con los datos obtenidos en este ensayo. Sin embargo, cabe señalar que las variaciones pueden ocurrir como resultado del propio material genético utilizado, así como de las variaciones en el peso específico de los frutos.

Los frutos de tomates del tipo Cereza son redondos con diámetro longitudinal menor o igual al transversal, mientras que los del tipo Grape son frutos oblongos con diámetro longitudinal mayor que el diámetro transversal y, por lo tanto, no pueden cumplir las mismas reglas de clasificación. 
Tabla 4. Interacción entre factores sistema y tamiz (Sist*Tam) para las variables número de frutos clasificados por tamiz y peso medio de los frutos en cada tamiz para la variedad Grape de tomate.

\begin{tabular}{|c|c|c|c|}
\hline \multirow{2}{*}{\multicolumn{2}{|c|}{$\begin{array}{l}\text { Interacción } \\
\text { Sist*Tam }\end{array}$}} & \multicolumn{2}{|c|}{ Grape Dolcetto } \\
\hline & & $\mathrm{N}^{\circ}$ frutos tamiz ${ }^{-1}$ & Peso Medio ( $\left.\mathrm{g}_{\text {fruto }}-1\right)$ \\
\hline \multirow{5}{*}{ Canaletas } & 1 & $7,00 \mathrm{C}$ & $13,34 \mathrm{~A}$ \\
\hline & 2 & $206,22 \mathrm{~A}$ & $8,73 \mathrm{~B}$ \\
\hline & 3 & $240,44 \mathrm{~A}$ & $5,74 \mathrm{C}$ \\
\hline & 4 & $28,67 \mathrm{C}$ & $3,04 \mathrm{D}$ \\
\hline & 5 & $2,00 \mathrm{C}$ & $0,86 \mathrm{E}$ \\
\hline \multirow{5}{*}{ Macetas } & 1 & $5,56 \mathrm{C}$ & $13,26 \mathrm{~A}$ \\
\hline & 2 & $144,78 \mathrm{~B}$ & $8,72 \mathrm{~B}$ \\
\hline & 3 & $216,67 \mathrm{~A}$ & $5,68 \mathrm{C}$ \\
\hline & 4 & $26,00 \mathrm{C}$ & $2,95 \mathrm{D}$ \\
\hline & 5 & $1,89 \mathrm{C}$ & $0,90 \mathrm{E}$ \\
\hline \multicolumn{2}{|l|}{ Media } & 87,92 & 6,32 \\
\hline \multicolumn{2}{|l|}{$\mathrm{CV} \%$} & 38,19 & 15,62 \\
\hline
\end{tabular}

Medias seguidas de misma letra en la columna no difieren estadísticamente entre sí pela prueba de Tukey $(\mathrm{p}<0,05)$.

Tabla 5. Clasificación de frutos de dos variedades de tomates según el diámetro de las perforaciones, clase, peso medio, número de frutos evaluados y sus porcentajes correspo ndientes a cada tamiz de clasificación.

\begin{tabular}{|c|c|c|c|c|c|c|}
\hline Variedad & Tamiz & $\begin{array}{l}\text { Diámetro perforaciones } \\
\qquad(\mathrm{mm})\end{array}$ & Clase & $\begin{array}{l}\text { Peso medio fruto } \\
(\mathrm{g})\end{array}$ & $\begin{array}{l}\mathrm{N}^{\circ} \text { frutos } \\
\text { evaluados }\end{array}$ & $\begin{array}{c}\% \text { de frutos } \\
\text { tamiz }^{-1}\end{array}$ \\
\hline \multirow{5}{*}{ Cereza } & 1 & mayor que 35 & Gigante & $>18$ & 185 & $2,23 \%$ \\
\hline & 2 & entre 30 - 35 & Grande & entre $13-18$ & 2849 & $34,39 \%$ \\
\hline & 3 & entre 25 - 30 & Mediano & entre 8 - 13 & 4148 & $50,07 \%$ \\
\hline & 4 & entre $20-25$ & Pequeño & entre $5-8$ & 608 & $7,34 \%$ \\
\hline & 5 & menor que 20 & Desecho & $<5$ & 494 & $5,96 \%$ \\
\hline \multirow{5}{*}{ Grape } & 1 & mayor que $25 \times 50$ & Gigante & $>12$ & 173 & $2,15 \%$ \\
\hline & 2 & entre $20-25 \times 50$ & Grande & entre $8-12$ & 3159 & $39,32 \%$ \\
\hline & 3 & entre $15-20 \times 50$ & Mediano & entre 5 - 8 & 4114 & $51,21 \%$ \\
\hline & 4 & entre $10-15 \times 50$ & Pequeño & entre $2-5$ & 492 & $6,12 \%$ \\
\hline & 5 & menor que $10 \times 50$ & Desecho & $<2$ & 95 & $1,18 \%$ \\
\hline
\end{tabular}

Por este motivo, específicamente para los frutos del tipo Grape, no se han encontrado referencias de clasificación de frutos, tanto en estándares nacionales como internacionales, que tengan en cuenta la característica del fruto (diámetro longitudinal mayor que el transversal). Así, este es el primer estudio que propone una metodología de clasificación de frutos para tomates del tipo Grape.

Independientemente de la variedad o tipo de fruto, es posible llevar a cabo la clasificación de los frutos por clases de acuerdo con su tamaño y peso medio, ya que las variables son dependientes entre sí, teniendo en cuenta la regresión lineal positiva significativa $(p<0,001)$ entre el tamaño de los frutos y sus pesos medios. Para ambas variedades, los coeficientes de determinación fueron $\mathrm{R}^{2}=0,93$. Souza et al. (2012) observaron una correlación positiva entre los caracteres de producción y calidad de los frutos de tomates de mesa, así como entre los caracteres de peso medio y diámetro de los frutos. De esta manera se puede demostrar que existe una relación entre las variables de tamaño y peso medio de los frutos de minitomates, con el fin de utilizar las variables para efectos de clasificación.

Los pesos mínimos aceptados por los mercados y que están dentro de las normas adecuadas de 
comercialización para los frutos del tipo Cereza deben ser de hasta $8 \mathrm{~g}$, mientras que para los frutos del tipo Grape hasta 5 g. Es decir, teniendo en cuenta la clasificación propuesta por este trabajo, los frutos comprendidos dentro de las clases de frutas gigantes, medianas y grandes para ambas variedades.

\section{Conclusiones}

La variedad, el sistema de cultivo y el deshojado influye en el tamaño de los frutos de tomate de diámetro pequeño. El deshojado disminuye la cantidad de frutos clasificados como grandes para las dos variedades analizadas, Cereza y Grape. El sistema de canales favorece la producción de frutos clasificados como grandes de la variedad Grape. Los tamices 1, 2 y 3 son adecuados para la clasificación de frutos teniendo en cuenta las normas de aceptación del mercado de consumo. Es posible clasificar frutos de tomates con el uso de tamices específicos para cada variedad.

\section{Literatura Citada}

Alvarenga, M.A.R.

2013. Tomate: produção em campo, casa de vegetação $e$ hidroponia. 2.ed., Ed. Universitária de Lavras. Lavras, Brasil. 455 p.

Beckles, D.M.

2012. Factors affecting the postharvest soluble solids and sugar content of tomato (Solanum lycopersicum L.) fruit. Postharvest Biology Technology, 63: 129-140.

Caron, V.C.; Tessmer M.A.; Mello S.C.; Jacomino A.P. 2013. Quality of mini tomatoes harvested at two maturity stages and kept chilled in three packages. Horticultura Brasileira, 31 (2): 279-286.

CEAGESP.

2003. Normas de classificação do tomate. CEAGESP. São Paulo, SP. 2 p.

Chamarro, J.L.

1995. Anatomia y fisiologia de la planta. In F. Nuez, editor, El cultivo del tomate. Mundi-Presa, Madrid, ESP. p. 43-91. CODEX.

2006. Codex Alimentarius Commission. FAO, Rome, ITA. $4 \mathrm{p}$.

Cruz, C.D.

2013. GENES: a software package for analysis in experimental statistics and quantitative genetics. Acta Scientiarum Agronomy, 35 (3): 271-276.

Fernandes, C.; Corá, J.E.; Braz, L.T.

2007. Classificação de tomate-cereja em função do tamanho e peso dos frutos. Horticultura Brasileira, 25 (2): 275-278.

Kanyomeka, L.; B. Shivute.

2005. Influence of pruning on tomato production under controlled environments. Agricultura Tropica Et Subtropica, 38 (2): 79-83.

Kim, S.E.; Lee, M.Y.; Lee, M.H.; Sim, S.Y.; Kim, Y.S.

2014. Optimal management of tomato leaf pruning in rockwool culture. Horticulture Environment and Biotechnology, 55 (6): 445-454.
Mazuela, P.A.; Cepeda, B.; Cubillos, V.

2012. Effect of grafting and the biostimulant Fartum ${ }^{\circledR}$ on production and quality in cherry tomatoes. Idesia, 30 (3): 77-81.

Mello, S.C.

2016. Cultivo de minitomates em substrato. In Oliveira, J.L.B.; R.B. Minuzzi, editores, Avanços no cultivo hidropônico de hortaliças de folhas e frutos. Tribo da Ilha, Florianópolis, SC. p. 58-65.

Menezes, J.B.C., Costa, C.A., Sampaio, R.A., Catão, H.C.R.M., Guilherme, D.O., Martinez, R.A.S.

2012. Fruit production and classification of four cherry tomato genotypes under an organic cropping system. Idesia, 30 (3): 29-35.

Peil, R.M.N.; Albuquerque Neto, A.A.R.; Rombaldi, C.V.

2014. Densidade de plantio e genótipos de tomateiro cereja em sistema fechado de cultivo em substrato. Horticultura Brasileira, 32 (2): 234-240.

Radin, B.; Lisboa, B.B.; Matzenauer, R.; Bardin, N.A.

2008. Avaliação da desfolha na produtividade do tomateiro. Pesquisa Agropecuária Gaúcha, 14 (1): 27-31.

Rocha, M.Q.; Peil, R.M.N.; Cogo, C.M.

2010. Rendimento do tomate cereja em função do cacho floral e da concentração de nutrientes em hidroponia. Horticultura Brasileira, 28 (4): 466-471.

Silva, L.J.; Milagres, C.C.; Silva, D.J.H.; Nick, C.; Castro, J.P.A. 2011. Basal defoliation and their influence in agronomic and phytopathological traits in tomato plants. Horticultura Brasileira, 29 (3): 377-381.

Souza, L.M.; Melo, P.C.T.; Luders, R.R.; Melo, A.M.T.

2012. Correlations between yield and fruit quality characteristics of fresh market tomatoes. Horticultura Brasileira, 30 (4): 627-631.

UNECE.

2012. FFV-36: concerning the marketing and commercial quality control of tomatoes. UNITED NATIONS. New York and Geneva, USA. 8 p. 
